\title{
A Preliminary Study on Various Off-line Hand Written Signature Verification Approaches
}

\author{
Meenakshi S Arya \\ Department of Computer Engineering and Information \\ Technology \\ Jaypee University of Information Technology \\ Waknaghat, P.O. Dumehar Kandaghat, \\ Solan-173 215 \\ Himachal Pradesh, INDIA.
}

\author{
Vandana S Inamdar \\ Department of Computer Engineering and \\ Information Technology \\ College of Engg. Pune, \\ Wellesely Rd, Shivajinagar, \\ Pune-411 005. \\ Maharashtra, INDIA.
}

\begin{abstract}
Biometrics can be classified into two broad categoriesbehavioral (signature verification, keystroke dynamics, etc.) and physiological (iris characteristics, fingerprint, etc.). Handwritten signature is amongst the first few biometrics to be used even before the advent of computers. Signature verification is widely studied and discussed using two approaches [5]. On-line approach uses an electronic tablet and a stylus connected to a computer to extract information about a signature and takes dynamic information like; pressure, velocity, etc whereas in offline approach stable dynamic variations are not used for verification purpose. Offline systems are more applicable and easy to use in comparison with on-line systems in many parts of the world however it is considered more difficult than on-line verification due to the lack of dynamic information. The paper presents a survey of off-line signature verification approaches being followed in different areas. This being a nascent area under research, the survey covers some of the examples of the ways
\end{abstract}

\section{Categories and Subject Descriptors Category: A [GENERAL LITERATURE] A.1 [INTRODUCTORY AND SURVEY] \\ Subject Descriptors: Signature verification}

\section{General Terms}

Security and Verification.

\section{Keywords}

offline signature verification, template matching, hidden markov models, spectrum -based approach

\section{INTRODUCTION}

Signature verification is an authentication method that uses the dynamics of a person's handwritten signature measure and analyzes the physical activity of signing, such as the stroke order, the pressure applied and the speed. Some systems may also compare visual images of signatures, but the core of a signature biometric system is behavioral, i.e. how it is signed rather than visual, i.e. the image of the signature.Each person has a unique handwritten signature. The way a person signs their name or writes a letter can be used to prove a person's identity. A pasted bitmap, a copy machine or an expert forger may be able to duplicate what a signature looks like, but it is virtually impossible to duplicate the timing changes in $\mathrm{X}, \mathrm{Y}$ and $\mathrm{Z}$ (pressure). The natural motion of the original signer would be required to repeat the patterns. Even though there will always be slight variations in a person's handwritten signature, the consistency created by natural motion and practice over time generates a recognizable pattern that makes the handwritten signature suitable for biometric identification. Inspite of being natural and intuitive this technology however has certain advantages as well as disadvantages associated with it. Some of the advantages being [4]:

1) The signature is the most natural and generally established of all the ways in which we seek to confirm our identity.

2) The use of signature verification will minimise the disruption to accepted practices with respect to transactions where personal identity has to be authenticated

3) Measurement of signature characteristics is noninvasive (compare this with other potential techniques such as iris scanning) and has no negative or undesirable health connotations (as might be the case with, say, fingerprint checking, which is often considered to raise civil liberties issues and which, in use, involves direct physical contact with a possibly contaminated surface). This is potentially a most important practical issue, the significance of which should not be underestimated.

Disadvantages:

1) There are some inconsistencies to a person's signature.

2) Great variability can be observed in signatures according to country, age, time, habits, psychological or mental state, physical and practical conditions [2].

\section{BASIC NOTION OF SIGNATURE VERIFICATION}

Signatures are composed of special characters and flourishes and therefore most of the time they can be unreadable. Also intrapersonal variations and interpersonal differences make it necessary to analyse them as complete images and not as letters and words put together. As signatures are the primary mechanism both for authentication and authorization in legal transactions, the need for research in efficient auto-mated solutions for signature recognition and verification has increased in recent years [11].

Recognition is finding the identification of the signature owner. Verification is the decision about whether the signature is genuine 
or forgery. In an off-line signature verification system, a signature is acquired as an image. This image represents a personal style of human handwriting, extensively described by the graphometry [10]. In such a system the objective is to detect three types of forgeries, which are related to intra and inter-personal variability [8]. The first type, called random forgery, is usually represented by a signature sample that belongs to a different writer of the signature model (see Fig. 1b). The second one, called simple forgery, is represented by a signature sample with the same shape of the genuine writer's name (see Fig. 1c). The last type is the skilled forgery, represented by a suitable imitation of the genuine signature model (see Fig.1d).

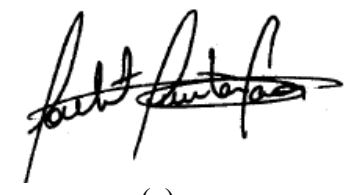

(a)

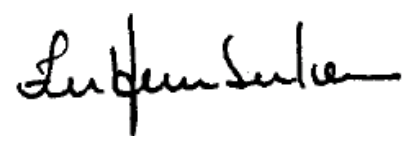

(c)

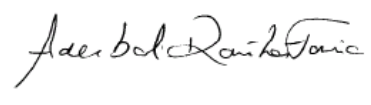

(b)

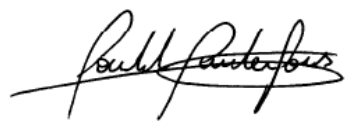

(d)
Fig. 1 Type of forgeries: (a) genuine signature; (b) random forgery; (c) simulated simple forgery; and (d) simulated skilled forgery.

Every type of forgery requests a different recognition approach. Methods based on Static approach are usually used to identify random and simple forgeries. The reason is that these methods have shown to be more suitable to describe characteristics related to the signature shape. The design of a signature verification system requires the solution of four problems; data acquisition, pre-processing, feature extraction and verification.

\subsection{Data acquisition and pre processing}

Signatures are scanned with 600dpi resolution, resulting in an average image size of $1000 * 250$ pixels. This resolution has shown to be necessary to correctly interpret the line crossings [12]. Signature preprocessing is a necessary step to improve the accuracy of the latter algorithms, and to reduce their computational needs. The following steps are done:

1) A noise filter (like median filter) is applied to remove the noise caused by the scanner.

2) The image is cropped, to the bounding rectangle of the signature.

3) Transformation from color to grayscale, and finally to black and white.

4) Thinning the black and white image results always in a huge information loss.

It is essential to select a thinning algorithm which gives a good abstraction of the original signature, with a low noise level.

\subsection{Feature extraction}

Features for offline signature verification using scanned images can be divided into three types $[13,14]$ :
1) Global features describe or identify the signature as a whole. They are extracted from every pixel that lies within a rectangle circumscribing the signature. These features do not reflect any local, geometrical, or topological properties of the signature, but include transformations, [15,16] series expansions [17], image gradient analysis[18]etc. Although global features are easily extractable and insensitive to noise, they are dependent upon the position alignment and highly sensitive to distortion and style variations.

2) Statistical features that are derived from the distribution of pixels of a signature, e.g. statistics of high gray-level pixels to identify pseudo-dynamic characteristics of signatures. This technique includes the extraction of high pressure factors with respect to vertically segmented zones (for example, upper, middle and lower zones) [19] and the ratio of signature width to short- or long-stroke height [20]. The statistical features take some topological and dynamic information into account and consequently can tolerate minor distortions and style variations.

3) Geometrical and topological features that describe the characteristic geometry and topology of a signature and thereby preserve the signatures global and local properties, e.g. local correspondence of stroke segments to trace signature [21]. Geometrical and topological features have a high tolerance to distortion and style variations, and they can also tolerate a certain degree of translation and rotation variations.

\section{APPROACHES TO SIGNATURE VERIFICATION}

\subsection{Template Matching Approach}

A process of pattern comparison, and is often called "template matching" [23]. Deng [39] developed a system that uses a closed contour tracing algorithm to represent the edges of each signature with several closed contours. The curvature data of the traced closed contours are decomposed into multiresolutional signals using wavelet transforms. The zero crossings corresponding to the curvature data are extracted as features for matching. A statistical measurement is devised to decide systematically which closed contours and their associated frequency data are most stable and discriminating. Based on these data, the optimal threshold value which controls the accuracy of the feature extraction process is calculated. Matching is done through dynamic time warping.

Fang et al.[34] proposed two methods for the detection of skilled forgeries using template matching. One method is based on the optimal matching of the one-dimensional projection profiles of the signature patterns and the other is based on the elastic matching of the strokes in the two-dimensional signature patterns. Given a test signature to be verified, the positional variations are compared with the statistics of the training set and a decision based on a distance measure is made. Both binary and grey-level signature images are tested. The average verification error rate of $18.1 \%$ was achieved when the local peaks of the vertical projection profiles of grey-level signature images were used for matching and with the full estimated covariance matrix incorporated [13].

Verification performance is affected by the variation of signature stroke widths and a registered signature selected from a set of reference samples in off-line signature verification using a pattern 
matching. Katsuhiko Ueda in [24] proposed the modified pattern matching method, which is independent of signature stroke width and the selection method of a registered signature for Japanese signature verification. Experimental results showed that the proposed methods improve the verification performance.

\subsection{Neural networks approach}

The main reasons for the widespread usage of neural networks (NNs) in pattern recognition are their power (the sophisticated techniques used in NNs allow a capability of modeling quite complex functions) and ease of use (as NNs learn by example it is only necessary for a user to gather a highly representative data set and then invoke training algorithms to learn the underlying structure of the data). The signature verification process parallels this learning mechanism. There are many ways to structure the NN training, but a very simple approach is to firstly extract a feature set representing the signature (details like length, height, duration, etc.), with several samples from different signers. The second step is for the $\mathrm{NN}$ to learn the relationship between a signature and its class (either "genuine" or "forgery"). Once this relationship has been learned, the network can be presented with test signatures that can be classified as belonging to a particular signer. NNs therefore are highly suited to modeling global aspects of handwritten signatures.

Alan McCabe et al. [25] proposed a method for verifying handwritten signatures by using $\mathrm{NN}$ architecture. Various static (e.g., height, slant, etc.) and dynamic (e.g., velocity, pen tip pressure, etc.) signature features are extracted and used to train the NN. Several Network topologies are tested and their accuracy is compared. The resulting system performs reasonably well with an overall error rate of $3.3 \%$ being reported for the best case.

Another method for off-line signature verification uses hough transform to detect stroke lines from signature image[27]. The Hough transform (general Radon transform) is used to extract the parameterized Hough space from signature skeleton as unique characteristic feature of signatures. In this method, the Back Propagation Neural Network is used as a tool to evaluate the performance of the proposed method. The system has been tested with 70 test signatures from different persons revealing the recognition rate of $95.24 \%$.

Rasha Abbas in his earlier research investigated the suitability of using backpropagation neural networks for the purpose of offline signature verification however later on in [2] the suitability of using multilayered feed forward neural network was investigated.

\subsection{Hidden Markov models approach}

Hidden Markov Model (HMM) is one of the most widely used models for sequence analysis in signature verification. Handwritten signature is a sequence of vectors of values related to each point of signature in its trajectory. Therefore, a well chosen set of feature vectors for HMM could lead to the design of an efficient signature verification system [3]. These Models are stochastic models which have the capacity to absorb the variability between patterns and their similarities. In HMM stochastic matching (model and the signature) is involved. This matching is done by steps of probability distribution of features involved in the signatures or the probability of how the original signature is calculated. If the results show a higher probability than the test signatures probability, then the signatures is by the original person, otherwise the signatures are rejected. The correct choice of the model topology in HMM is fundamental to obtain a satisfactory result in the learning and verification phase. There are various topologies for the HMM models, each of which adapt to one particular characteristic. For the discrete models, two factors are predominant. The first is the number of states to be used and the second is the number of transitions between these states [27]. Besides the choice of the HMM-topology, the probability density function modeling of the HMMs is the most important part in order to design the most appropriate models for the verification task.

Yacoubi et al. [27] proposed a basic and robust system for the verification of static or offline signatures. For this, simple features were used so that the performance of the learning and verification system, using HMM, could be confirmed. AERs of $0.46 \%$ and $0.91 \%$ are reported in his experiment.

Justino et al. [6] in his work presented a robust system for off-line signature verification using simple features, different cell resolutions and multiple codebooks in an HMM framework. The simple and random forgery error rates have shown to be low and close of each other. An FRR of $2.83 \%$ and an FAR of $1.44 \%$, $2.50 \%$, and $22.67 \%$ are reported for random, casual, and skilled forgeries, respectively.

A method for the automatic recognition of off-line handwritten signatures using both global and local features is described in [28]. As global features the method uses the envelope of the signature sequenced as polar coordinates; and as local features points located inside the envelope that describe the density or distribution of signature strokes are used. Each feature is processed as a sequence by a hidden Markov Model (HMM) classifier. The results of both classifiers are linearly combined obtaining a recognition ratio of $95.15 \%$.

Coetzer et al[40]developed a system that automatically authenticates offline handwritten signatures using the discrete Radon transform (DRT) and a hidden Markov model (HMM). Given the robustness of the algorithm and the fact that only global features are considered, the system achieves an equal error rate (EER) of $18 \%$ when only high-quality forgeries (skilled forgeries) are considered and an EER of $4.5 \%$ in the case of only casual forgeries.

Other applications of HMMs in signature verification can be found in $[9,29,30]$.

\subsection{Statistical approach}

Using statistical knowledge, the relation, deviation, etc between two or more data items can easily be found out. Strictly speaking, to find out the relation between some set of data items we generally follow the concept of Correlation Coefficients. In general statistical usage refers to the departure of two variables from independence. To verify an entered signature with the help of an average signature, which is obtained from the set of, previously collected signatures, this approach follows the concept of correlation to find out the amount of divergence in between them.

A Bayesian model for off-line signature verification involving the representation of a signature through its curvature is developed by McKeague[31]. The prior model makes use of a spatial point 
process for specifying the knots in an approximation restricted to a buffer region close to a template curvature, along with an independent time warping mechanism. In this way, prior shape information about the signature can be built into the analysis. The observation model is based on additive white noise superimposed on the underlying curvature. The approach is implemented using Markov chain Monte Carlo (MCMC) algorithm and applied to a collection of documented instances of Shakespeare's signature.

The algorithm proposed in [3] has the flexibility of choosing the number of signatures, i.e., no_of_Sign for testing purpose to generate a signature as Avg_Sign containing the specialized mean features set from the test signatures set. After collecting the signatures for testing, the algorithm converts them into a set of 2D arrays of binary data values- 0 and 1 . From these binary arrays using statistical methods of calculating expected mean an average data set. The Recognition scheme is based on extensive Statistical Analysis of Correlation Coefficient between bivariate data set. In implementation of proposed algorithm to constant factors carry major impact on the validity of the method and the strength of the verification lies in the efficiency of selection of these constant parameters, namely Avg_Sign, Threshold Value and decision value.

\subsection{Structural or syntactic approach}

The key idea in structural and syntactic pattern recognition is the representation of patterns by means of symbolic data (signatures etc.) structures such as strings, trees, and graphs. In order to recognize an unknown pattern (forged signature), its symbolic representation is compared with a number of prototypes stored in a database. Structural features use modified direction and transition distance feature (MDF) which extracts the transition locations and are based on the relational organization of low-level features into higher- level structures. The Modified Direction Feature (MDF) [32] utilizes the location of transitions from background to foreground pixels in the vertical and horizontal directions of the boundary representation of an object.

Nguyen et al [1] presents a new method in which structural features are extracted from the signature's contour using the (MDF) and its extended version: the Enhanced MDF (EMDF) and further two neural network-based techniques and Support Vector Machines (SVMs) are investigated and compared for the process of signature verification. The classifiers were trained using genuine specimens and other randomly selected signatures taken from a publicly available database of 3840 genuine signatures from 160 volunteers and 4800 targeted forged signatures. A distinguishing error rate (DER) of $17.78 \%$ was obtained with the SVM whilst keeping the false acceptance rate for random forgeries (FARR) below $0.16 \%$.

Another very interesting method proposed by Ferrer et al.[33] calculates geometric features of a signature in fixed-point arithmetic for offline verification. The proposed features are then checked with different classifiers, such as Hidden Markov Models, Support Vector Machines, and the Euclidean distance verifier. The results show that HMM works slightly better than SVM and the distance Euclidean verifier, but, bearing in mind that the SVM and Euclidean distance-based verifiers can be programmed in a fixed-point microprocessor, the results encourage us to follow the SVM research line in order to built a smart card capable of detecting a simple forgery.
In his paper Fang et al.[34] describes that from a collection of genuine and skilled forgery signatures, it is observed that although skilled forgery signatures are very similar to genuine ones on a global scale, they are generally less smooth and natural on a detailed scale than the genuine ones, especially for those skilled forgery signatures which consist of cursive graphic patterns. A smoothness index is derived from such signatures. This is combined with other global shape features and used for verification. Furthermore, for those graphic type signatures with long cursive lines, the forgery signatures are generally less smooth and natural than the genuine ones on a detailed scale. Hence for those graphic type signatures, the smoothness of the cursive segments of the strokes can be used as one of the features for verification. The method proposed extracts a smoothness feature from graphic type signatures and combines it with other useful global geometric features to use it for off-line signature verification.

\subsection{Wavelet- based approach}

In general, the multi-resolution wavelet transform can decompose a signal into lowpass and highpass information. The highpass information usually represents features that contain sharper variations in time domain. Wavelet theory $[36,37]$ is used to decompose a curvature-based signature into a multi-resolution signal. If the whole signature curves are matched, it's very hard to distinguish the genuine signatures and the forged ones effectively, because the signature curves are very complex and changeful, even the genuine signatures of the same person have very large differences.

A novel approach to off-line signature verification is proposed by Wei Tian et al. [35] Both static and pseudodynamic features are extracted as original signal, which are processed by Discrete Wavelet Transform (DWT) and converted into stable features in each sub-band which can enhance the difference between a genuine signature and its forgery. During the training phase, the proposed fuzzy net is trained with genuine signatures only. The signatures with the maximal ratio of the mean value of the similarity to the standard deviation are selected as the training samples from a set of genuine signatures. The verification scheme is achieved by combining the proposed fuzzy net output in each sub-band level. The entire system was tested by using two databases of English and Chinese signatures, and the average error rates of $12.57 \%$ and $13.96 \%$ were obtained, respectively.

Another novel approach to off-line handwritten signature verification is proposed in [38]. The method uses a wavelet-based transformation to extract the inflections of the signature curves by using different scale wavelet transforms in the curvature signature signals transformation. After analysis, proper scale is selected. The zero-crossings points are extracted and are taken as the inflections of the signature. Then the signature curves are divided into several parts, i.e. the strokes, according to these inflections. The distance between two corresponding strokes can be measured with Dynamic Time Warping algorithm. In the end, the training algorithm of the signature verification system and the verification method of the signatures are also introduced. The experimental results show that this method is superior to those methods that match the whole signature curves. It can detect the forged signatures from the genuine ones successfully. 
Deng et al. [39] describe a wavelet-based off-line handwritten signature verification system capable of automatically identifying useful and common features which consistently exist within different signatures of the same person . The system starts with a closed-contour tracing algorithm. The curvature data of the traced closed contours are decomposed into multiresolutional signals using wavelet transforms. Then the zero-crossings corresponding to the curvature data are extracted as features for matching. Moreover, a statistical measurement is devised to decide systematically which closed contours and their associated frequency data of a writer are most stable and discriminating. Based on these data, the optimal threshold value which controls the accuracy of the feature extraction process is calculated. The proposed approach can be applied to both on-line and off-line signature verification systems. Experimental results show that the average success rates for English signatures and Chinese signatures are $92.57 \%$ and $93.68 \%$, respectively.

\section{COMPARISON}

Each type of forgery requires a different verification approach. Hence it becomes mandatory to compare these approaches with respect to various levels of forgeries.

Template matching is suitable for rigid matching to detect genuine signatures however these methods are not very efficient in detecting skilled forgeries.

Neural networks are among the most commonly used classifiers for pattern recognition problems. This approach offers a significant advantage that each time we want to add a set of signatures (a new person) to the systems database; we only have to train three new small neural networks (one for each set of features) and not the entire neural network. This approach gives very promising results with extremely low FAR and FRR.

Methods based on the statistical approach are generally used to identify random and simple forgeries. The reason for this is that these methods have proven to be more suitable for describing characteristics related to the signature shape. For this purpose, the graphometry-based approach has many features that can be used, such as calibration, proportion, guideline and base behaviors. In addition, other features have been applied in this approach, like pixel density, pixel distributions. However, static features do not describe adequately the handwriting motion. Therefore, it is not enough to detect skilled forgery.

When using HMMs for signature verification, we can find that the simple and random forgery error rates have shown to be low and close to each other, but the type II error rate in skilled forgery signatures are high.

Structural techniques are suitable for detecting genuine signatures and targeted forged signatures however, this approach is exhaustive due to demand for large training sets and computational efforts.

Spectrum analysis finds its application not only in offline signature verification but online signature verification too. It is used to decompose a curvature- based signature into a multiresolution format and can be applied to even symbolic languages like Chinese and Japanese along with English.

\section{CONCLUSION}

Of all biometric technologies, whether biological or nonbiological, Signature Recognition offers most potential in terms of adaptability and implementation. This holds true from a number of perspectives i.e. ease of use, low implementation cost and the ease of embedding the system in an organization, without excessively disrupting or affecting existing operations. In this paper an attempt has been made to analyze various methods for offline signature verification and hence provide a literary platform for the enhancement of the existing approaches as well as development of new ones in this field.

\section{REFERENCES}

[1] Vu Nguyen; Blumenstein, M.; Muthukkumarasamy V.; Leedham G., "Off-line Signature Verification Using Enhanced Modified Direction Features in Conjunction with Neural Classifiers and Support Vector Machines", in Proc. $9^{\text {th }}$ Int Conf on document analysis and recognition, vol 02, pp. 734-738, Sep 2007.

[2] Rasha Abbas and Victor Ciesielski, "A Prototype System for Off-line Signature Verification Using Multilayered Feed forward Neural Networks," February 1995.

[3] Bhattacharyya Debnath, Bandyopadhyay Samir Kumar, Das, Poulami, Ganguly Debashis, Mukherjee Swarnendu, "Statistical approach for offline handwritten signature verification", Journal of Computer Science March 01, 2008.

[4] MI C. Fairhurst, "Signature verification revisited: promoting practical exploitation of biometric technology", Electronics \& communication engineering journal, December 1997.

[5] N. G. See, O.H.Seng, "A Neural network approach for offline signature verificaiton”, IEEE International Conference on Speech and Image Technologies for Computing and Telecommunications, pp.770-773, 1993.

[6] Edson J. R. Justino, Flávio Bortolozzi and Robert Sabourin , "Off-line Signature Verification Using HMM for Random, Simple and Skilled Forgeries", in International Conference on Document Analysis and Recognition, vol. 1, pp. 105-110, Seattle, Wash, USA, 2001.

[7] ] Q. Yingyong, B. R. Hunt, "Signature Verification Using Global and Grid Features", Pattern Recognition - vol. 22, no. 12, Great Britain (1994), 1621--1629.

[8] Drouhard, J.P., R. Sabourin, and M. Godbout, “A neural network approach to off-line signature verification using directional PDF", Pattern Recognition, vol. 29, no. 3, (1996), 415--424.

[9] G. Rigoll, A. Kosamala, “A Systematic Comparison Between On-line and Off-line methods for Signature Verification with HMM", $14^{\text {th }}$ ICPR, vol. 2, pp. 1755-1757, Australia, 1998.

[10] Edson J. R. Justino, A. El Yacoubi, F. Bortolozzi and R. Sabourin, "An Off-Line Signature Verification System Using HMM and Graphometric Features", DAS 2000, 4th IAPR International Workshop on Document Analysis Systems, Rio de Janeiro, Brazil, (2000), pp 211--222. 
[11] Emre Özgündüz,Tülin Şentürk and M. Elif Karslıgil, “OffLine Signature Verification and Recognition by Support Vector Machine".

[12] Zsolt Kertész1, Bence Kővári2, "Off-Line Signature Verification using Feature Based Image Registration".

[13] B. Fang, C.H. Leung, Y. Y. Tang, K. W. Tse, P. C. K. Kwok, and Y. K. Wong, "Off-line signature verification by the tracking of feature and stroke positions," Pattern Recognition, vol. 36, pp. 91-101, 2003.

[14] S. Lee and J. C. Pan, "Offline tracing and representation of signatures”, IEEE Trans. Syst. Man Cybern. 22 (1992) $755\{771\}$.

[15] M. E. Munich and P. Perona, "Visual identification by signature tracking", IEEE Trans.Patt. Anal. Mach. Intell. 25(2) (2003).

[16] W. F. Nemcek and W. C. Lin, "Experimental investigation of automatic signature verification", IEEE Trans. Syst. Man Cybern. SMC-4 (1974) 121-126.

[17] C. C. Lin and R. Chellappa, "Classification of partial 2-D shapes using Fourier descriptors, IEEE Trans. Patt. Anal. Mach. Intell. 9 (1997) 696-690.

[18] R. Sabourin and R. Plamondon, "Preprocessing of handwritten signatures from image gradient analysis", in Proc. $8^{\text {th }}$ Int. Conf. Pattern Recognition, 1986, pp. 576-579.

[19] M. Ammar, Y. Yoshido and T. Fukumura, "A new effective approach for offline verification of signatures by using pressure features", in Proc. $8^{\text {th }}$ Int. Conf. Pattern Recognition, 1986, pp. 566-569.

[20] R. N. Nagel and A. Rosenfeld, "Computer detection of freehand forgeries”, IEEE Trans. Comput., 1997, pp. 895905.

[21] J. K. Guo, D. Doermann and A. Rosenfeld, "Local correspondence for detecting random forgeries", in Proc. Int. Conf. Document Analysis and Recognition, 1997, pp. 319323.

[22] J. Coetzer, B. M. Herbst and J. A. du Preez, "Offline Signature Verification Using the Discrete Radon Transform and a Hidden Markov Model", EURASIP Journal on Applied Signal Processing 2004:4, 559-571

[23] Stuart Inglis ,Ian H. Witten, “Compression-based Template Matching”, Proc. IEEE Data Compression Conference, pp. 106-115, Los Alamitos, CA, 1994.

[24] Katsuhiko Ueda, "Investigation of Off-Line Japanese Signature Verification Using a Pattern Matching”, Proc. of the $7^{\text {th }} I C D A R, 2003$.

[25] Alan McCabe, Jarrod Trevathan and Wayne Read, "Neural Network-based Handwritten Signature Verification", Journal of computers, vol. 3, no. 8, August 2008.

[26] Tonphong Kaewkongka, Kosin Chamnongthai and Bundit Thipakom, "Off-Line Signature Recognition using parameterized Hough Transform", $5^{\text {th }}$ International

[40] J. Coetzer, B. M. Herbst, J. A. du Preez, “Offline Signature Verification Using the Discrete Radon Transform and a
Symposium on Signal Processing and its Applications, Brisbane, Australia, 22-25 August, 1999.

[27] Edson J. R. Justino, Abdenaimel Yacoubi, Flaviob Ortolozzi and Roberts Abourin, "An Off-Line Signature Verification System Using Hidden Markov Model and Cross-Validation", in IEEE International Workshop on Neural Networks for Signal Processing, pp. 859-868, Sydney, Australia, December 2000.

[28] Jose' A. Sanchez, Carlos M. Travieso, Itizar G. Alonso and Miguel A. Ferrer, "Handwritten signatures recognizer by its envelope and strokes layout using HMM's", in proc. IEEE $35^{\text {th }}$ Int carnahan Conf on Security Technology", vol 1, pp. 267-271, Oct 2001.

[29] G. Rigoll,et al, "A Comparison between continuous and Discrete Density Hidden Markov Models for Cursive Handwriting Recognition”, Proc. IEEE-ICPR, Vienna, vol. 2, pp. 205-209, 1997.

[30] L.Yang, et al, "Applications of HMMs for Signature Verification”, Pattern Recognition, vol. 28, Issue 2, pp. 161-170, Feb, 1995.

[31] Ian W. McKeague, "A statistical model for signature verification”, May 14, 2004.

[32] M. Blumenstein, X. Y. Liu, and B. Verma, "A Modified Direction Feature for Cursive Character Recognition," in International Joint Conference on Neural Networks, pp. 2983-2987, 2004.

[33] Miguel A. Ferrer, Jesu's B. Alonso, and Carlos M. Travieso, "Offline Geometric Parameters for Automatic Signature Verification Using Fixed-Point Arithmetic", IEEE Transactions On Pattern Analysis And Machine Intelligence, vol. 27, No. 6, June 2005.

[34] B. Fang, Y.Y. Wang, C.H. Leung, Y.Y. Tang, P.C.K. Kwok, K.W. Tse and Y.K. Wong, "A Smoothness Index Based Approach for Off-line Signature Verification”, 2000.

[35] Wei Tian, Yizheng Qiao and Zhiqiang Ma, “A New Scheme for Off-line Signature Verification Using DWT and Fuzzy Net", $8^{\text {th }}$ ACIS International Conference on Software Engineering, Artificial Intelligence, Networking, and Parallel/Distributed Computing.

[36] S. G. Mallat, "Zero-crossings of a Wavelet Transform," IEEE Trans.Inform. Theory 37(4), pp. 1019-1033, 1991.

[37] S. G. Mallat, "A Theory for Multiresolution Signal Decomposition: The Wavelet Representation," IEEE Trans. Pattern Anal. Mach. Intell. 11(7), pp. 674-693, 1989.

[38] Xiufen Ye, Weiping Hou and Weixing Feng, "Off-line Handwritten Signature Verification with Inflections Feature", Proc. of the IEEE International Conference on Mechatronics \& Automation, Canada, July 2005.

[39] Peter ShaoHua Deng, et al, "Wavelet-based off-line handwritten signature verification", Computer vision and image understanding, Vol.76, Issue 3, pp. 173-190, Dec 1999.

Hidden Markov Model", EURASIP Journal on Applied Signal Processing vol. 4, pp. 559-571,2004. 